\title{
It works in my hands
}

\section{Bruce Donoff}

\section{Dean, Harvard School of Dental Medicine, Boston, USA}

All improvements require change, but not all change is improvement. If we continue to behave as if evidence is bad for patient care we will continue to foster the clinical adage of 'if it works well in my hands it must be good'.

How many times have we all heard this claim by clinicians citing their best evidence? I frequently hear it with regard to procedure-oriented care from cardiac surgery to dentistry. Most recently, in my own specialty of oral and maxillofacial surgery, it was invoked surrounding the use of a particular type of rigid fixation for mandibular fractures ${ }^{1,2,3}$. A new method may be too techniquesensitive to be a true advance in treatment of patients by a practitioner not trained in the methods. It was because of a similar behaviour of clinicians in my department that I embarked upon a road that has led me to be an advocate for evidence-based dentistry.

Our journal club met monthly, led by house staff with numerous attending surgeons present. It seemed that a review of the literature was always a time to bring out the most important anecdotes of each and every surgeon present. One did it his way because his chief had said it was best ten years before. Another used an alternative because some piece of equipment was not always available. The important point is that, clinical pearls aside (and there were plenty of valid and relevant pearls), the majority of recommendations were based purely on clinical experience. Although it is recognised that the development of clinical judgement is a critically important part of residency training, the time had come to add real depth and substance to our journal clubs.

Then I came upon a wonderful series of articles called "How to read the
Clinical Journals" in the Canadian Medical Association Journal in 1981. ${ }^{4-8}$ Little did I know that Drs Sackett, Haynes, Tugwell, and Trout were the early exponents of a movement that would become known as evidence-based medicine (EBM). The use of the five papers in journal review sessions became a highlight of each resident's experience. Anecdotally, it also led to 'belief' only in papers published in certain journals.

A recent editorial in Journal of Dental Research emphasised the research-related and practice-related of evidencebased dentistry (EBD). ${ }^{9}$ 'The practice of EBD requires the blending of research knowledge with provider experience'. There is nothing inherent in EBD that is threatening to the wisdom of clinical experience and sound judgement. It does require that the appropriate question be asked for the clinical problem for which a decision is sought. It also requires the need for high-quality evidence. ${ }^{10}$ Recognising the uncertainty of everything we do in clinical medicine and dentistry, it appears most clinicians are not prepared to integrate best evidence into clinical practice. Many practitioners feel that EBD cannot be used to enhance, but only interfere with the professional judgement of the dentist. This would seem to be primarily because action based upon evidence is considered judgement by other than the practitioner. This is as unrealistic and unreasonable as assuming that everything in dentistry and medicine can be based upon evidence only. Why do patients get second and third opinions? This is both a reflection of human nature and the uncertainty of decision making in clinicians not only agreeing about a diagnosis, but also in recommending a treatment.

Chambers in a recent paper entitled 'The Roles of Evidence and the Baseline in Dental Decision Making' offers an explanation for some of the tension in our profession surrounding evidencebased dentistry. ${ }^{11} \mathrm{He}$ suggests that it relates back to the two components of evidence-based practice; one researchrelated, the other practice-related. The availability of evidence from randomised controlled clinical trials, (which yield the highest level of association) or evidence based upon studies in which the strength of association must be determined is at best moderate for dentistry. What is often forgotten in integrating the two components of EBD is that even the best studies of treatment need to consider relevance. In the original MacMaster series on 'How to read the Literature', two criteria used in determining useful from useless or even harmful therapy are: 'were the patients similar to your own?', and 'is the therapeutic manoeuvre possible in your practice?' These points need to be considered even in the gold standard randomised double-blinded clinical trial. Evidence-based dentistry should not be confused with protocol-based practice. It should not be reserved for unusual circumstances. The repertoire of the dentist may be based upon expert knowledge and skills, but experience can lead to comfort that ignores evidence for change. How else to explain disregard of evidence concerning the use of sealants and current recommen- 
dations regarding the removal of wisdom teeth? In dentistry, as in any surgical discipline, the use of sham procedures as a placebo pose an enormous ethical dilemma.

How can dentistry participate in evidence-based practice? Although the quality of the data may not be as strong as with placebo-controlled studies, studies without randomisation, multicentre case-controlled studies, or comparisons between intervention and no intervention studies, and the critical evaluation of case reports, can still help decision making. The important point is not to accept new procedures until sufficient supportive data become available. Equally as a corollary we should not assume that all baseline 'current practices' (to use Chambers' terms) are accurate. The differences between two groups of data look totally different if the baseline ordinate is zero or if it is not. The trick is that baseline suppression magnifies any differences between groups. All improvements require change, but not all change is improvement. If we continue to behave as if evidence is bad for patient care we will continue to foster the clinical adage of 'if it works well in my hands it must be good'.

1. lizuka $T$, Lindquiest $C$. Rigid internal fixation of mandibular fractures: an analysis of 270 fractures treated using the AO/ASIF method. Int J Oral Maxillofac Surg 1992; 21:65-69.

2. Kearns O, Perrot D, Kaban L. Rigid fixation of mandibular fractures: does operator experience reduce complication? J Oral Maxillofac Surg 1994; 52:226-232.

3. Kearns O. Discussion of a paper Treatment of Mandibular Fractures with rigid fractures using rigid osteosynthesis: Using the AO system. J Oral Maxillofac Surg 1997; 55:1406-1407.

4. Department of Clinical Epidemiology and Biostatistics, McMaster University, Hamilton, Ontario. How to read clinical journals: I. Why to read them and how to start reading them critically. Can Med J 1981; 124:555-558.

5. Department of Clinical Epidemiology and Biostatistics, McMaster University,
Hamilton, Ontario. How to read clinical journals: II. To learn about a diagnostic test. Can Med J 1981; 124:703-710.

6. Department of Clinical Epidemiology and Biostatistics, McMaster University, Hamilton, Ontario. How to read clinical journals: III. To learn the clinical course and prognosis of disease. Can Med J 1981; 124:869-872.

7. Department of Clinical Epidemiology and Biostatistics, McMaster University, Hamilton, Ontario. How to read clinical journals: IV. To determine etiology or causation. Can Med J 1981; 124:985-990.

8. Department of Clinical Epidemiology and Biostatistics, McMaster University, Hamilton, Ontario. How to read clinical journals: $V$. To distinguish useful from useless or even harmful therapy. Can Med J 1981; 124:1156-1162.

9. Bader E, Ismail A, Clarkson J. Evidencebased Dentistry and the Dental Research Community. J Dent Res 1999; 78:14801483.

10. Niederman R, Badovinac K. Tradition-based Dental Care and Evidence-based Dental

Care. J Dent Res 1999; 78:1288-1291.

11. Chambers DW. The Roles of Evidence and the Baseline in Dental Decision Making. J Am Coll Dent 1999; 66:60-68. 